\title{
Préparez-vous !
}

\section{J.C. Debongnie}

(C) Springer-Verlag 2009

Le récent congrès de Fès, manifestation annuelle de notre association, était l'expression de la richesse dont Francis Klotz parle dans l'éditorial du précédent numéro du $J A H G$. Une organisation impeccable, de nombreuses communications de qualité, un intérêt inattendu pour les ateliers organisés par et pour cette revue. Tout y était. Tout manifestait le dynamisme de nos amis marocains, dynamisme depuis la fondation de l'association. C'est un exemple pour le futur : chaque congrès pourrait être l'occasion d'un feu d'artifice du pays organisateur, dont les travaux oraux (ou autres) présentés lors de congrès pourraient être publiés dans la revue.

L'article sur les hémorragies digestives ulcéreuses, dans ce numéro, est un bon exemple de ce que nous souhaitons pour cette revue : un article original sur un sujet où il existe peu de données africaines, concernant un problème commun et fréquent en hépatogastroentérologie et qui permet d'ouvrir la question à d'autres travaux. Il y a peu de données africaines : sur près de 40000 articles recensés dans Medline concernant GI bleed (hémorragies gastrointestinales), 190 sont associées au mot « Africa », et si l'on exclut les hémorragies variqueuses et les articles d'Afrique anglophone (surtout sud-africains), il ne reste que très peu d'articles non casuistiques. Le principal nous vient du Cameroun, publié en 1990 dans Médecine tropicale, entre autres, par notre président actuel C. Tzeuton, et concerne 172 cas d'hémorragie digestive haute examinés en endoscopie.

Que nous apprend l'étude publiée dans ce numéro ? La surprise principale vient du bon pronostic : récidive hémorragique dans $14,5 \%$ des cas, mortalité de 3,7\%. En l'absence d'hémostase endoscopique, le pronostic est deux fois meilleur que dans les séries occidentales ! Le pronostic serait-il avant tout fonction de l'âge et des comorbidités ? Ce point pourrait être confirmé par de nouvelles analyses d'autres séries prospectives africaines. L'hémostase endoscopique permettrait de réduire sans doute la nécessité d'interventions chirurgicales, réalisées chez près de $10 \%$ des patients. Les recommandations actuelles proposent l'usage d'inhibiteurs de la pompe à protons par voie intraveineuse à forte dose, traitement très coûteux. Une publication canadienne récente montre l'efficacité du traitement intraveineux, à doses normales. Les études futures montreront peut-être l'efficacité de fortes doses orales, ce qui a été le cas dans l'étude de Fès, et qui est sans doute à recommander en Afrique.

Il est important de traduire les résultats d'études thérapeutiques en notions cliniques. C'est ce que fait le NNT (number needed to treat) que nous pouvons appliquer à l'hémorragie digestive. Les publications montrent, d'une part, que l'usage de rofécoxib - inhibiteur des COX2 (produit coûteux) plutôt que celui de naproxen - réduit le risque relatif (RRR) d'hémorragie digestive de $60 \%$, d'autre part, que l'hémostase endoscopique réduit de $50 \%$ le risque de récidive hémorragique, ce qui paraît comparable. Et pourtant, dans le premier cas, ce risque passe de 2,3 à $1 \%$, soit une réduction absolue de $1,3 \%$, dans l'autre de 40 à $20 \%$, soit une réduction absolue de $20 \%$. Dans le premier cas, il faudra traiter 77 patients (NNT) pour éviter une hémorragie $(1 / 0,0013$ cas $1 /$ réduction absolue du risque), dans le second cas, il ne faut traiter que cinq patients $(1 / 0,2)$ pour éviter une récidive hémorragique. Extrapolons à l'article de Benajah: supposons que l'hémostase endoscopique réduise le risque de chirurgie de 10 à $2 \%$ (réduction absolue de $8 \%$ ). Il faudrait traiter 12 patients pour éviter une intervention chirurgicale.

O. Nouel a présenté à Fès les résultats d'une enquête multicentrique française extra-universitaire sur l'hémorragie digestive réalisée en France, publiée aux États-Unis. Un questionnaire est en préparation pour l'Afrique. Préparezvous! 